\title{
Design and Fabrication of Silicon Photonic Crystal Optical Waveguides
}

\author{
Marko Lončar, Theodor Doll, Jelena Vučković, and Axel Scherer
}

\begin{abstract}
We have designed and fabricated waveguides that incorporate two-dimensional (2-D) photonic crystal geometry for lateral confinement of light, and total internal reflection for vertical confinement. Both square and triangular photonic crystal lattices were analyzed. A three-dimensional (3-D) finite-difference timedomain (FDTD) analysis was used to find design parameters of the photonic crystal and to calculate dispersion relations for the guided modes in the waveguide structure. We have developed a new fabrication technique to define these waveguides into silicon-on-insulator material. The waveguides are suspended in air in order to improve confinement in the vertical direction and symmetry properties of the structure. High-resolution fabrication allowed us to include different types of bends and optical cavities within the waveguides.
\end{abstract}

Index Terms-Finite-difference time-domain (FDTD) methods, nanooptics, optical device fabrication, photonic bandgap (PBG) materials, photonic crystals (PCS), photonic crystal waveguides.

\section{INTRODUCTION}

$\mathbf{P}$ HOTONIC crystals [1], [2] (PCs), two- and three-dimensionally periodic structures with high refractive index contrast, have recently become very interesting for the manipulation of light. The existence of a photonic bandgap, a frequency range in which propagation of light is prevented in all directions, makes photonic bandgap (PBG) structures very useful in applications where spatial localization of light is required. For example, by forming defects in a host PBG structure, we can form localized electromagnetic states, and "trap the light." Ideally, by making a three-dimensional (3-D) photonic crystal we can control propagation of light in all three dimensions [3]-[5]. Since fabrication of 3-D PC structures is still a difficult process, a more appealing approach is based on the use of lower dimensional photonic crystals to achieve confinement of light in three dimensions. A structure that has recently attracted a lot of attention is a dielectric slab perforated with a two-dimensional (2-D) PC lattice [6]-[10]. In such a structure, light is controlled in the vertical direction by the means of total internal reflection (TIR) (due to the refractive index contrast of high index core and low index cladding), and in the lateral direction by distributed Bragg reflection (DBR), due to the presence of the 2-D PC. Recently,

Manuscript received December 23, 1999; revised June 28, 2000. This work was supported in part by the AFOSR under Contract AFS-49620-98-1-01447.

M. Lončar, J. Vučković, and A. Scherer are with the Department of Electrical Engineering, California Institute of Technology, MS 136-93, Pasadena, CA 91125 USA (e-mail: loncar@its.caltech.edu).

T. Doll was with the Department of Physics, California Institute of Technology, MS 136-93, Pasadena, CA 91125 USA. He is now with the Faculty of Electrical Engineering, Ilmenau Technical University, Ilmenau 98693, Germany.

Publisher Item Identifier S 0733-8724(00)09101-5. there were reports on characterization of both active [11] and passive [12] devices based on this system.

The idea of using line defects in photonic crystals to guide light was proposed by Meade et al. [13]. Mekis et al. [14] have developed the theoretical foundation of understanding $90^{\circ}$ bends in the 2-D photonic crystals. Experimental confirmation of this theoretical work in the millimeter-wave range using long alumina rods was provided by Lin et al. [15]. The disadvantage of using photonic lattices constructed from dielectric rods surrounding an air waveguide is that there is no mechanism for low-loss vertical confinement of the light within the waveguide at optical frequencies. A more favorable geometry is the one introduced above, a thin dielectric slab perforated with holes, within which a line defect of missing holes can be defined. 3-D theoretical analysis [16], [17] and qualitative experimental characterization [18]-[20] of light guiding within waveguides made in the photonic crystal slabs have been recently reported.

In this paper, we present the theoretical analysis and fabrication of waveguides that incorporate 2-D photonic crystal geometries for lateral confinement of light, and total internal reflection for vertical confinement.

\section{THEORETICAL ANALYSIS}

We start with a theoretical analysis of the band structure for both square and triangular lattice photonic crystals in order to find design parameters, such as interhole spacing $(a)$, radius of holes $(r)$, and thickness of the slab $(d)$, that will give us a bandgap centered around interesting $1.5-\mu \mathrm{m}$ wavelength range. Full 3-D analysis is required since the waveguide incorporates vertical confinement of light. In contrast to the infinite 2-D case, finite-thickness slabs can support modes with higher order vertical oscillations, and, if the slab is made too thick, the presence of these modes can result in closing of the bandgap [9]. Therefore, the thickness of the slab is a critical parameter in this structure and needs to be modeled. In addition, 2-D analysis of vertically extended structure (infinitely thick slab) would result in band diagrams that are shifted toward the lower frequencies. The reason for that is the fact that in the case of the slab waveguide guided modes are not completely confined in the slab, but also extend into the air. Therefore, the energy of the guided mode will be higher and the bands will be at higher frequencies than in the case of 2-D waveguide with infinitely long holes.

The structure that we analyze first is a silicon slab patterned with square or triangular lattice of holes and surrounded by air (Fig. 1). Three-dimensional final-difference time-domain (3-D FDTD) scheme [21], [22] was used to calculate band diagrams 


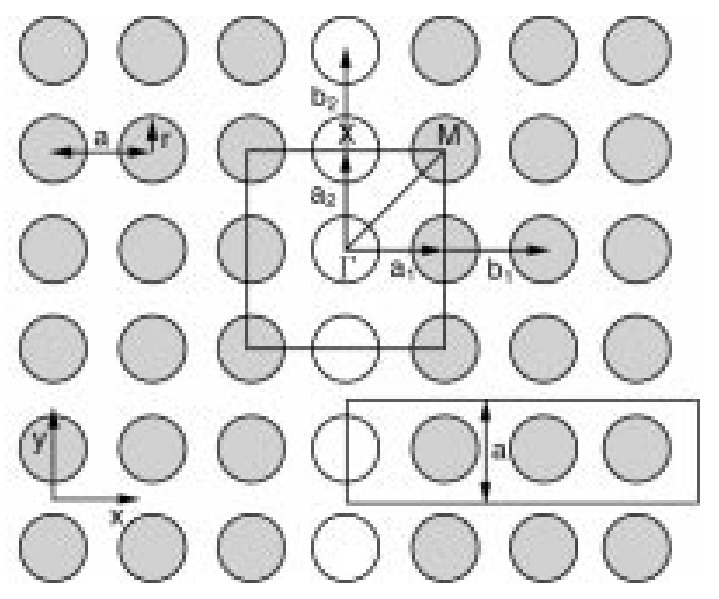

(a)

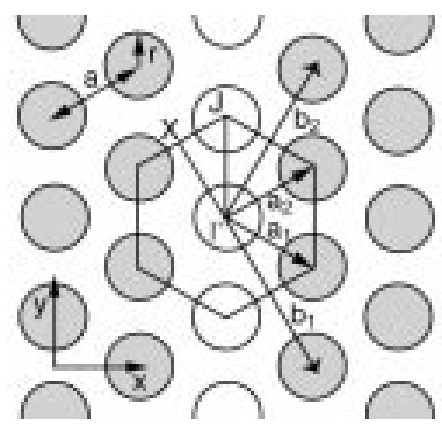

(b)

Fig. 1. (a) Si slab perforated with 2-D square lattice of holes (top view). Lattice vectors are $a_{1}=a \cdot(1,0,0)$ and $a_{2}=a \cdot(0,1,0)$. Reciprocal lattice vectors are $b_{1}=(2 \pi / a) \cdot(1,0,0)$ and $b_{2}=(2 \pi / a) \cdot(0,1,0)$. Without loss of generality, interhole spacing $a$ in the figure is chosen to be $a=\pi / a$ (dimensionless units), in order to conveniently show reciprocal lattice vectors. The square represents the first Brillouin zone (BZ), and $\Gamma, X$, and $M$ are the high symmetry points with coordinates (in reciprocal space): $\Gamma=(0,0,0), X=(0, \pi / a, 0), M=(\pi / a, \pi / a, 0)$. Empty circles represent location where the waveguide will be defined by removing the entire row of holes. (b) Si slab perforated with 2-D triangular lattice of holes (top view). Lattice vectors are $a_{1}=a \cdot(\sqrt{3} / 2,-1 / 2,0)$, and $a_{2}=a \cdot(\sqrt{3} / 2,1 / 2,0)$. Reciprocal lattice vectors are $b_{1}=(4 \pi / a \sqrt{3}) \cdot(1 / 2,-\sqrt{3} / 2,0)$, and $b_{2}=(4 \pi / a \sqrt{3}) \cdot(1 / 2, \sqrt{3} / 2,0)$. As explained in (a), we chose $a=4 \pi / 3 a$ in order to represent reciprocal and real-space lattice vectors in the same figure. The hexagon represents the first BZ. Coordinates of high symmetry points are: $\Gamma=(0,0,0), X=(2 \pi / a \sqrt{3}) \cdot(1 / 2,-\sqrt{3} / 2,0)$, and $J=(4 \pi / 3 a) \cdot(0,1,0)$.

for square and triangular [23] lattices. In both cases, we analyzed a lattice unit cell by applying appropriate boundary conditions to its sides: Bloch boundary conditions to all four sides perpendicular to the plane of the slab, Mur's absorbing boundary condition [24] (ABC) to the top boundary, and mirror boundary condition at the middle of the slab. In that way we reduce the computation time by analyzing only one half of the unit cell. By changing the type of the mirror symmetry we could select even (TE-like) or odd (TM-like) eigenmodes of the photonic crystal structure. The spatial resolution that we used to discretize our structures was 20 computational points per interhole spacing $(a=20)$. The thickness of the Si slab was equal to $d=0.55 a$ and the hole radius was $r=0.4 a$. The index of refraction of $\mathrm{Si}$ is assumed to be $n_{\mathrm{Si}}=3.5$. Mur's ABC was applied at a distance of $3 * a=60$ computational points away from the top surface of the slab (approximately one midgap wavelength in the air), yielding the computation box of $20 * 20 * 65$ cubical cells.

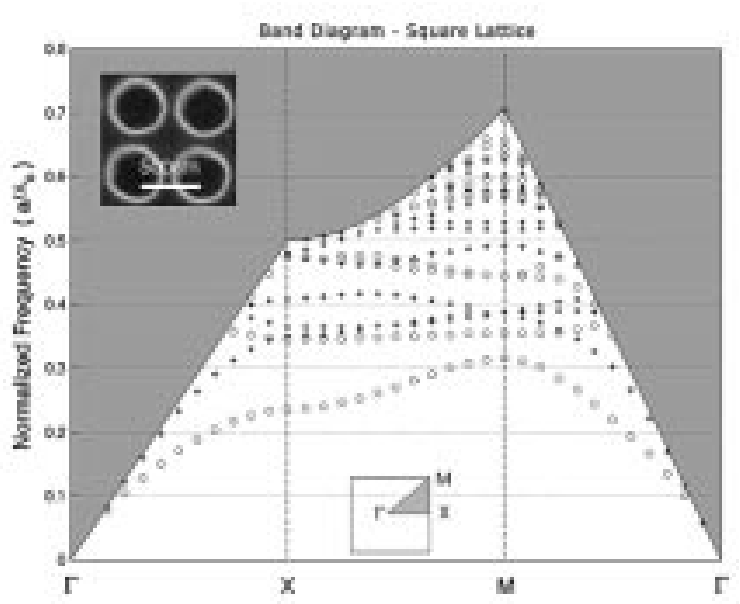

(a)

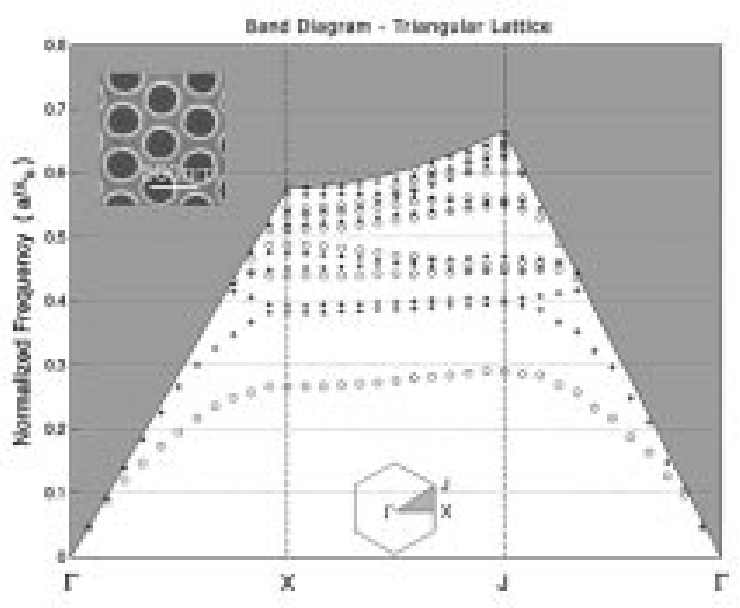

(b)

Fig. 2. Band diagrams of eigen modes of Si slab perforated with 2-D lattice of holes of (a) square and (b) triangular symmetry. The thickness of the slab is chosen to be $d=0.55 a$ and the hole radius is $r=0.4 a$, where $a$ is the interhole spacing. Even modes are represented with circles and odd with stars. Insets show the SEM micrographs of the fabricated photonic crystal structures (scale bar is $500 \mathrm{~nm}$ ), and first Brillouin zones with characteristic high symmetry points.

Band diagrams for the even (TE-like) and odd (TM-like) eigenmodes of the PC structure with square symmetry are shown in Fig. 2(a), and with triangular symmetry in Fig. 2(b). The $x$-axis represents different directions in the reciprocal lattice (see Fig. 1), and the $y$-axis normalized frequency in units of $a / \lambda=\omega a / 2 \pi c$, where $\lambda$ is the wavelength in the air. The solid line shown in the plots represents the light line-dispersion relation for the photons in the air having momentum as one on the $x$-axis. Modes with frequencies above the light line will leak energy into the air, and thus represent the loss mechanism of the waveguide.

As can be seen, in both structures the first-order bandgap is open for the guided modes of the slab (modes below the light line) that have an even symmetry (TE-like). However, it is important to notice that the Si slab perforated with 2-D photonic crystal does not have a full 3-D bandgap. Since in the third dimension (vertical direction) light is confined to the slab due to the total internal reflection, a bandgap is formed only for the guided modes, as mentioned above. Modes with frequencies 
TABLE I

Design Parameters of the Photonic Crystal Used in Order to HaVe BandGap Centered Around $\lambda=1.5 \mu \mathrm{m}$

\begin{tabular}{c|c|c|c|c|c|c|c|c}
\hline $\begin{array}{c}\text { Lattice } \\
\text { type }\end{array}$ & $\begin{array}{c}\text { Bandgap width } \\
\text { (start, end) } \\
{[\mathrm{a} / \lambda]}\end{array}$ & $\begin{array}{c}\text { Midgap } \\
\text { frequency } \\
\mathrm{f}_{0}[\mathrm{a} / \lambda]\end{array}$ & \multicolumn{3}{|c|}{$\begin{array}{c}\text { Lattice Parameters } \\
\text { Theory }\end{array}$} & \multicolumn{3}{c}{$\begin{array}{c}\text { Lattice Parameters } \\
\text { Fabricated Structure }\end{array}$} \\
\cline { 5 - 9 } & & & $\mathrm{a}=\mathrm{f}_{0} * \lambda$ & $\mathrm{r}=0.4 \mathrm{a}$ & $\mathrm{d}=0.55 \mathrm{a}$ & $\mathrm{a}$ & $\mathrm{r}$ & $\mathrm{d}$ \\
\hline Square & $\begin{array}{c}0.031 \\
(0.315,0.346)\end{array}$ & 0.33 & $496 \mathrm{~nm}$ & $198 \mathrm{~nm}$ & $272 \mathrm{~nm}$ & $500 \mathrm{~nm}$ & $200 \mathrm{~nm}$ & $280 \mathrm{~nm}$ \\
\hline Triangular & $\begin{array}{c}0.148 \\
(0.291,0.439)\end{array}$ & 0.365 & $547 \mathrm{~nm}$ & $219 \mathrm{~nm}$ & $301 \mathrm{~nm}$ & $530 \mathrm{~nm}$ & $208 \mathrm{~nm}$ & $300 \mathrm{~nm}$ \\
\hline
\end{tabular}

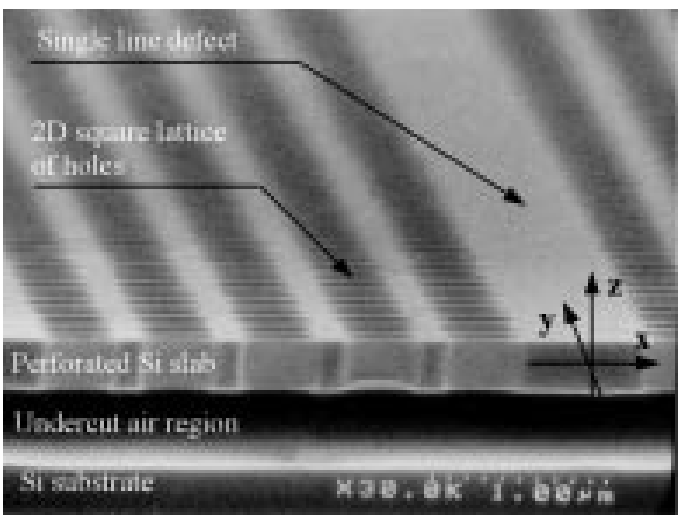

(a)

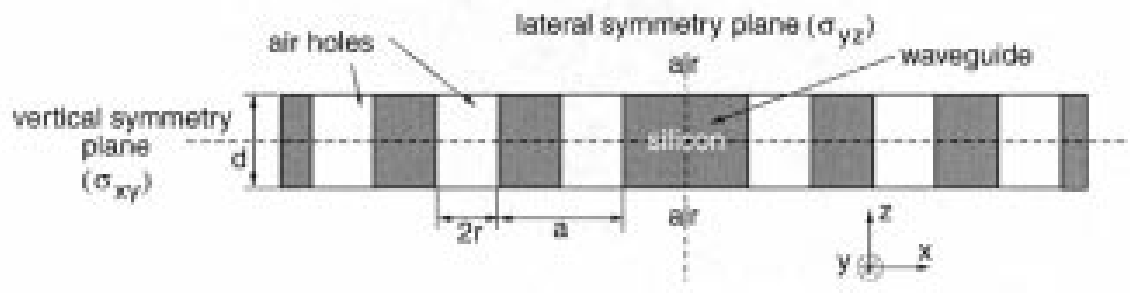

(b)

Fig. 3. (a) Cross section of the fabricated square lattice waveguide suspended in the air. (b) Schematic of the waveguide viewed end-on. Reflection symmetry planes are: $\sigma_{x y}$ in the middle of the slab (vertical symmetry plane), and $\sigma_{y z}$ in the center of the waveguide (lateral symmetry plane).

above the light line can couple to the continuum of the radiative modes, and therefore a complete bandgap does not exist [7]. As can be seen in Fig. 2, the triangular lattice has a much wider bandgap than the square lattice, a result of the greater symmetry and the smoother Brillouin zone in that geometry. If we take into account fabrication tolerances (size and shape of the holes, thickness of the slab, etc.) it is possible that in the fabricated square lattice structure the bandgap will be closed. Therefore, the triangular lattice is in many ways a more tolerant candidate for investigating the properties of the photonic crystal waveguides.

From Fig. 2 we can find the design parameters for each structure so that the bandgap is centered around wavelength of $\lambda=$ $1.5 \mu \mathrm{m}$. The results are summarized in the Table I. Once we find the value of interhole spacing (a), we can calculate the resolution of our FDTD algorithm (in nanometers). We find, using Table I, that the edge length of one computational cell is $24.8 \mathrm{~nm}$ in the case of square lattice, and $27.35 \mathrm{~nm}$ in the case of triangular lattice.

When we introduce a line defect into the photonic crystal by removing an entire line of holes from the 2-D crystal lattice (Figs. 1 and 3), we form the simplest photonic crystal wave- guide [1], [13], [14]. By forming such a defect, we are adding donor impurities to the photonic crystal, and as a result modes from the air band are pulled down into the bandgap and form defect states. Energy can now propagate only along this line defect, and this results in waveguiding of light. Propagation in the lateral direction is suppressed since the defect mode is within the bandgap of the photonic crystal, and propagation in the vertical direction is prevented since the mode is below the light line. In our case, waveguides are extending along $y$-axis in 3-D space (Fig. 3). Due to the periodicity of the lattice, the waveguides analyzed here have discrete translational symmetry with periodicity $a$. In Fig. 1(a), one half of the waveguide 1-D unit cell is represented with a rectangle of width $a$.

Single-line defect waveguides were modeled using the 3-D FDTD algorithm. Discretization was again 20 points per interhole spacing $(a=20)$. Since the waveguide has reflection symmetry in vertical $\left(\sigma_{x y}\right)$ and lateral $\left(\sigma_{y z}\right)$ direction, we could reduce computation time by analyzing only one quarter of the structure by applying mirror boundary conditions in the middle of the slab and in the center of the waveguide (Fig. 3). Bloch boundary conditions were applied in $y$-direction, at the boundaries of the waveguide unit cell. For analysis of the square lat- 


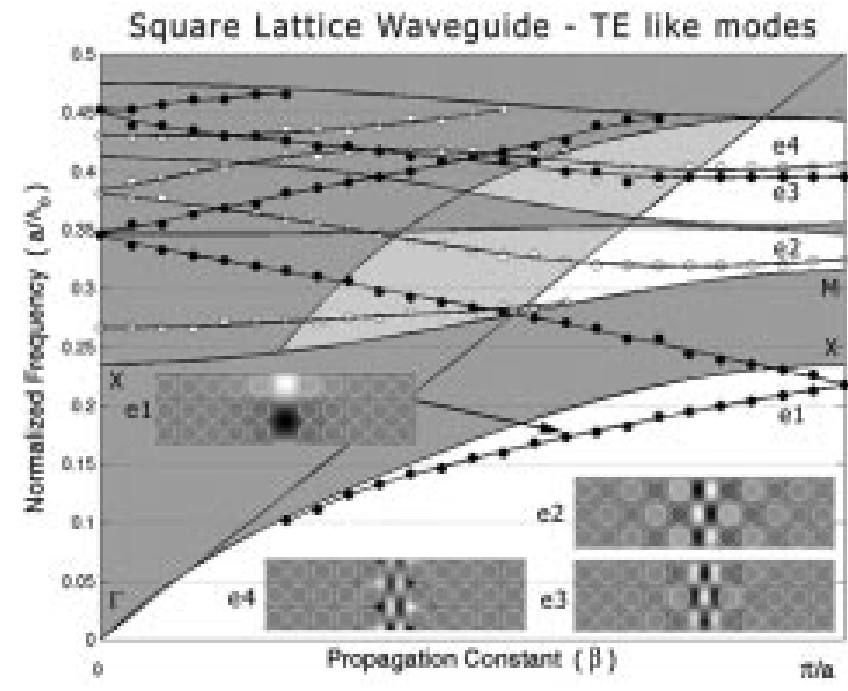

(a)

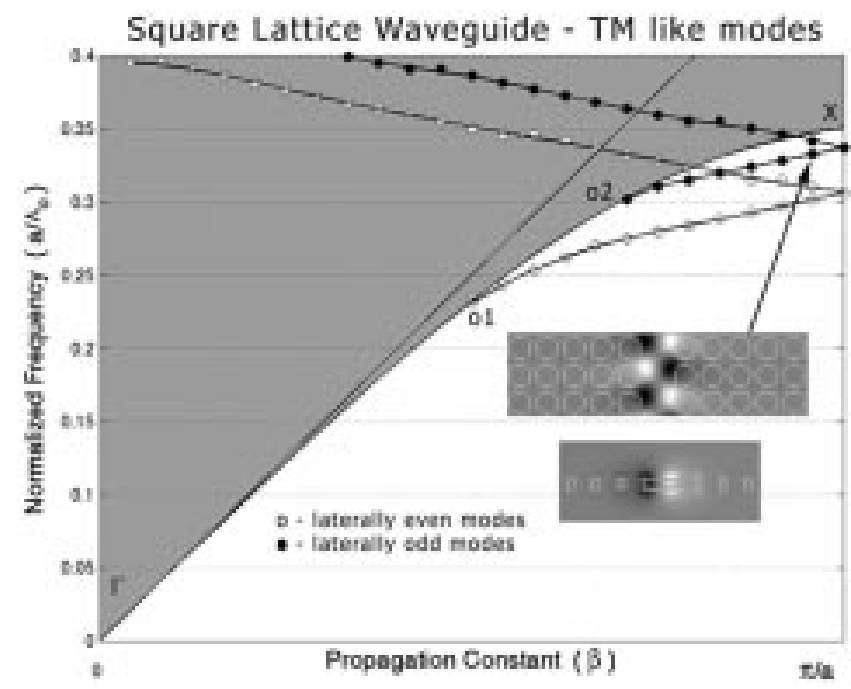

(b)

Fig. 4. Dispersion diagram for (a) vertically even (TE-like) and (b) vertically odd (TM-like) guided modes in the waveguide made as a single-line defect in a square PC lattice. Dispersion diagram is calculated for 25 different values of the propagation constant $k_{y}(\beta)$ in the interval $(0, \pi / a)$. Symmetry of the modes with respect to the lateral symmetry plane $\left(\sigma_{y z}\right)$ is indicated. Solid line from $(0,0)$ to $(\pi / a, 0.5)$ represents the light line. Regions where guiding can occur are shown in white color. Leaky modes are mapped out as well (modes in the gray regions). Modes are labeled e1-e4 for vertically even case (TE-like), and o1-o2 for vertically odd case (TM-like). Characteristic high symmetry points are also shown (dielectric band only). Insets show field patterns of (a) $B_{z}$ and (b) $E_{z}$ components in the middle of the slab ( $z$-slice) for different guided modes. In the case of TM-like guided modes (o1 and o2), the distribution of the $E$. component along the cross section of the waveguide is also shown. The intensity scale bar is shown in Fig. 6.

tice, Mur's ABCs were applied in the vertical direction, at a distance $3 * a=60$ computational units away from the surface of the waveguide, and in the lateral direction, after five layers of photonic crystal. Therefore, the computational domain consisted of $110 * 20 * 65$ cubic cells. The advantage of using absorbing boundary conditions over the supercell approach is that we can map out not only the guided modes of the structure, but also the leaky modes-modes that satisfy vertical resonance in the waveguide but lie above the light line. For analysis of the triangular PC lattice, Mur's ABCs were applied after eight layers

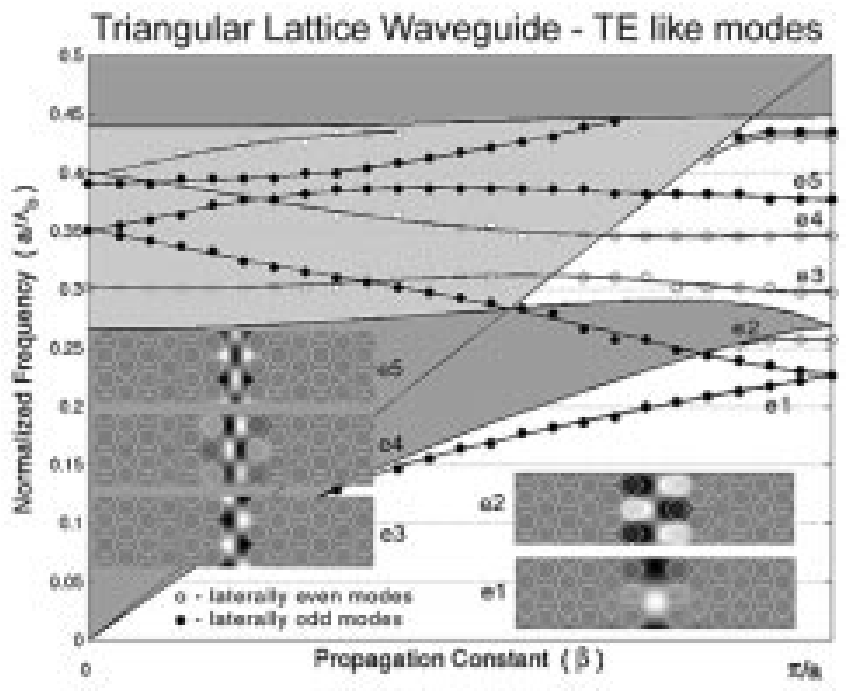

(a)

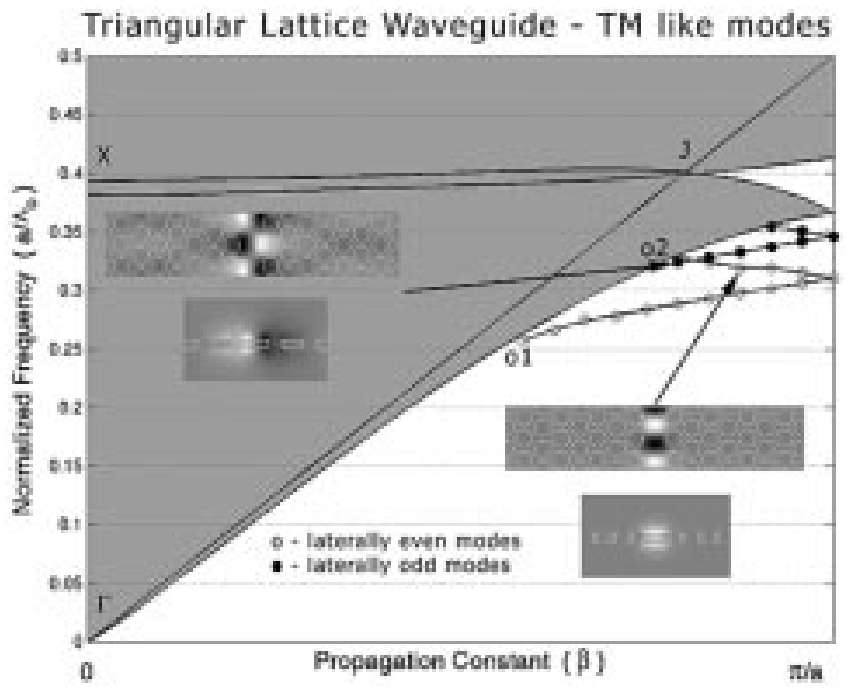

(b)

Fig. 5. Dispersion diagram for (a) vertically even (TE-like) and (b) vertically odd (TM-like) guided modes in the waveguide based on the triangular PC lattice. The light line, guiding regions, and leaky regions are labeled as in Fig. 4. Leaky modes are not shown in the case of TM-like modes. Insets show field patterns of (a) $B_{z}$ and (b) $E_{z}$ components in the middle of the slab ( $z$-slice). In the case of TM-like modes $(01,02)$, the distribution of the $E_{z}$ component along the cross section of the waveguide ( $\sigma_{x z}-$ plane) is shown as well (note that the cross sections for the $\mathrm{o} 1$ and $\mathrm{o} 2$ modes are taken at different positions along the waveguide). Field profile for $\mathrm{o} 2$ is taken near the cutoff and, therefore, the field penetrates the photonic crystal, as can be seen in the inset. The scale bar is shown in the Fig. 7.

of photonic crystal (in lateral direction). The other boundary conditions used were the same as in the case of the square lattice. Therefore, computational domain in the case of the triangular lattice consisted of $141 * 20 * 65$ cubic cells.

We present dispersion diagrams for guided modes of the single-line defect photonic crystal waveguide in Figs. 4 and 5. Symmetry of these modes with respect to the $\sigma_{y z}$-plane is indicated. The propagation constant $\beta$, also labeled as $k_{y}$, is on the $x$-axis of the diagram, and the normalized frequency in units of $a / \lambda$ is on the $y$-axis.

The direction of propagation of light in the case of the waveguide made in a square-lattice $\mathrm{PC}$ is along the $\Gamma X$ direction in 

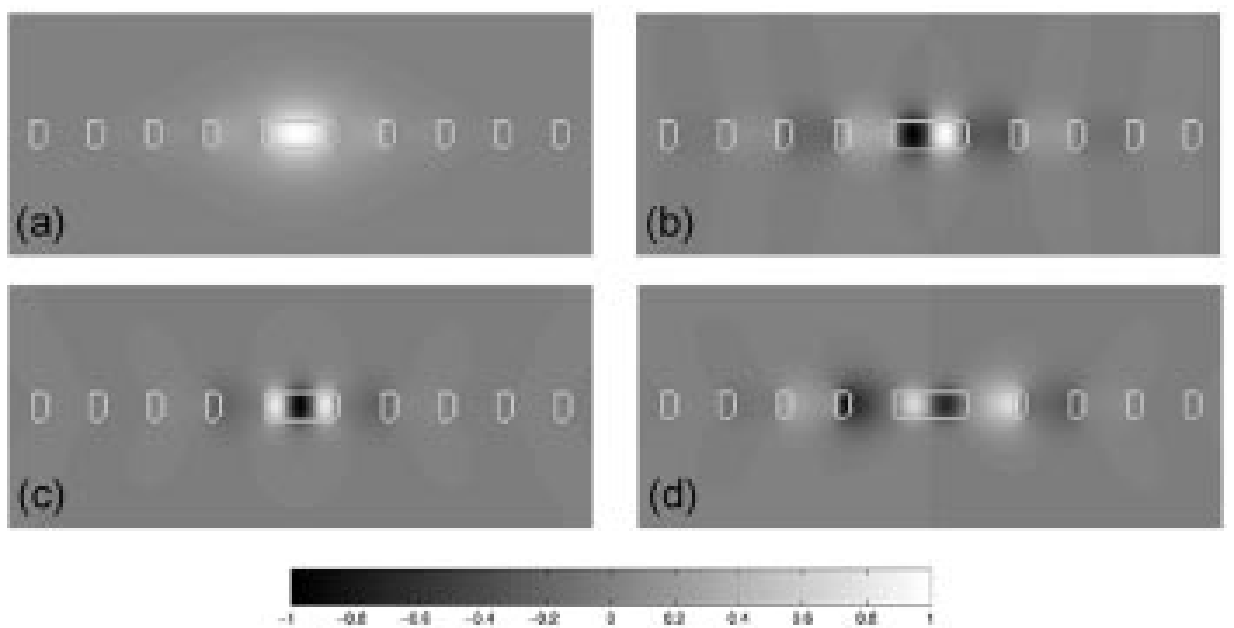

Fig. 6. Distribution of the $B_{z}$ component of the field along the cross section of the square-lattice-based waveguide ( $\sigma_{x z}$-plane) for different guided modes. Labeling is (mode \#, $\left.k_{y} \cdot(a / \pi), a / \lambda\right)$. (a) (e1, 15/24, 0.173), (b) (e2, 22/24, 0.320), (c) (e3, 22/24, 0.395), and (d) (e4, 22/24, 0.404). It can be seen that all the modes are fundamental in the vertical direction. The core of the waveguide appears as the rectangle in the center of the figure. Intensity scalebar is also shown.

the reciprocal space [Fig. 1(a)]. Due to the periodicity of the waveguide in the $y$ direction, $k_{y}$ can only take values in the interval $(0, \pi / a)$, or in terms of 2 -D PCs, any value from $\Gamma X$ direction. The procedure for finding dispersion diagram of guided modes, introduced in the [25], is as follows. We fix one value of $k_{y}$ by applying Bloch boundary conditions to the boundaries of the waveguide unit cell. Then, we evolve in time the initial field in the waveguide and store the field evolution in several low-symmetry points. We find the spectrum of these time evolutions by applying a fast Fourier transform. Peaks in the spectrum correspond to the characteristic modes of the structure [25]. However, not all of the modes that we find in this way are necessarily guided modes of the waveguide. We can detect modes of the PC slab, as well. Since guided modes can exist only in the region in $\left(k_{y}, a / \lambda\right)$ space where there are no photonic crystal modes, it is necessary to project all the bands of PC lattice from the Fig. 2(a) onto the $\Gamma X$ direction. In Figs. 4 and 5, the dark gray regions are regions where modes of photonic crystal slab exist. Those regions are obtained from Fig. 2, taking into account not only the guided modes shown there, but also the leaky modes. The light line is indicated with solid line from $(0,0)$ to $(\pi / a, 0.5)$. All modes above the light line, in the light gray region, will leak energy into the air, and modes that exist in the dark gray region will couple to the states of photonic crystal and leak energy into it. The regions where 3-D localization of light in the waveguide can occur are shown in white in both Figs. 4 and 5.

Analyzing Fig. 4, it becomes clear that there are two mechanisms of light confinement in the lateral direction.

First, guiding can occur if the mode is below all photonic crystal modes (modes e1, o1, and o2 in Fig. 4). These modes do not "see" the Si slab perforated with 2-D PC lattice as the PBG material, but rather as material with an effective refractive index $\left(n_{\text {eff }}\right)$ lower than the refractive index of $\mathrm{Si}$ core $\left(n_{\mathrm{Si}}=3.5\right)$. The mechanism of guiding for these modes is exactly the same as in the standard ridge waveguide - total internal reflection. Since they do not see periodicity of the $\mathrm{PC}$ there is no upper limit for the propagation constant $k_{y}(\beta)$, and therefore, at the zone boundary $\left(k_{y}=\pi / a\right)$, they are folded back into the first Brillouin zone of the waveguide.
Also, there is no bandgap opened at the zone boundary for these modes, another striking evidence of the fact that the modes are purely index-guided. When these modes cross the regions where the modes of the PC slab exist, they interact with them and leak energy into the PC. This is due to the fact that the modes of the PC slab extend outside the first BZ.

Another type of guiding occurs for the modes that are located in the bandgap (modes e2-e4 in Fig. 4). These modes are truly photonic crystal effect guided modes, and would not exist if there were no photonic crystal. They are pulled down, in the bandgap, from the air band and, therefore, we expect them to have the properties of that band. Since the bottoms of the air bands are flat (see band diagrams of the even modes in the $X M$ direction, Fig. 2), we expect these PC effect guided modes to have flat dispersion relations (Fig. 4). The flatness of the dispersion relation will result in small group velocity and inefficient transport of energy, the property that needs to be investigated in more details.

Field profiles of the $B_{z}$ (TE-like modes) and $E_{z}$ (TM-like modes) components of the field in the middle of the slab $\left(\sigma_{x y}\right.$-plane) are shown as insets in Fig. 4. Fig. 6 shows field distribution of the same components along the cross section of the waveguide ( $\sigma_{x z}$-plane) for several different modes. As we can see, all the modes are fundamental in the vertical direction, and have a different number of nodes in the lateral direction. In this work, we are interested in the PBG effect guided modes: mode e 2 in the first-order bandgap, and modes e 3 and e 4 in the second-order bandgap. Field profiles for these modes are taken for $k_{y}=\frac{22}{24} \frac{\pi}{a}$. For this value of $k_{y}$, mode e 2 approaches, in $\left(k_{y}, a / \lambda\right)$ space, modes of PC slab (gray region), couples to them and leaks energy into the photonic crystal. This can be observed in the inset e2 in Fig. 4(a) as the $B_{z}$ field pattern penetrating the photonic crystal. If we move away from the zone boundary, closer to the light line, the mode will start to radiate more energy into the air, and again will be lossy. If we want to excite the e2 mode with an external light source, light will also couple to the leaky e1 mode, since it exists in the same frequency range, and coupling efficiency will decrease. In addition, the dispersion diagram for the TM-like modes 

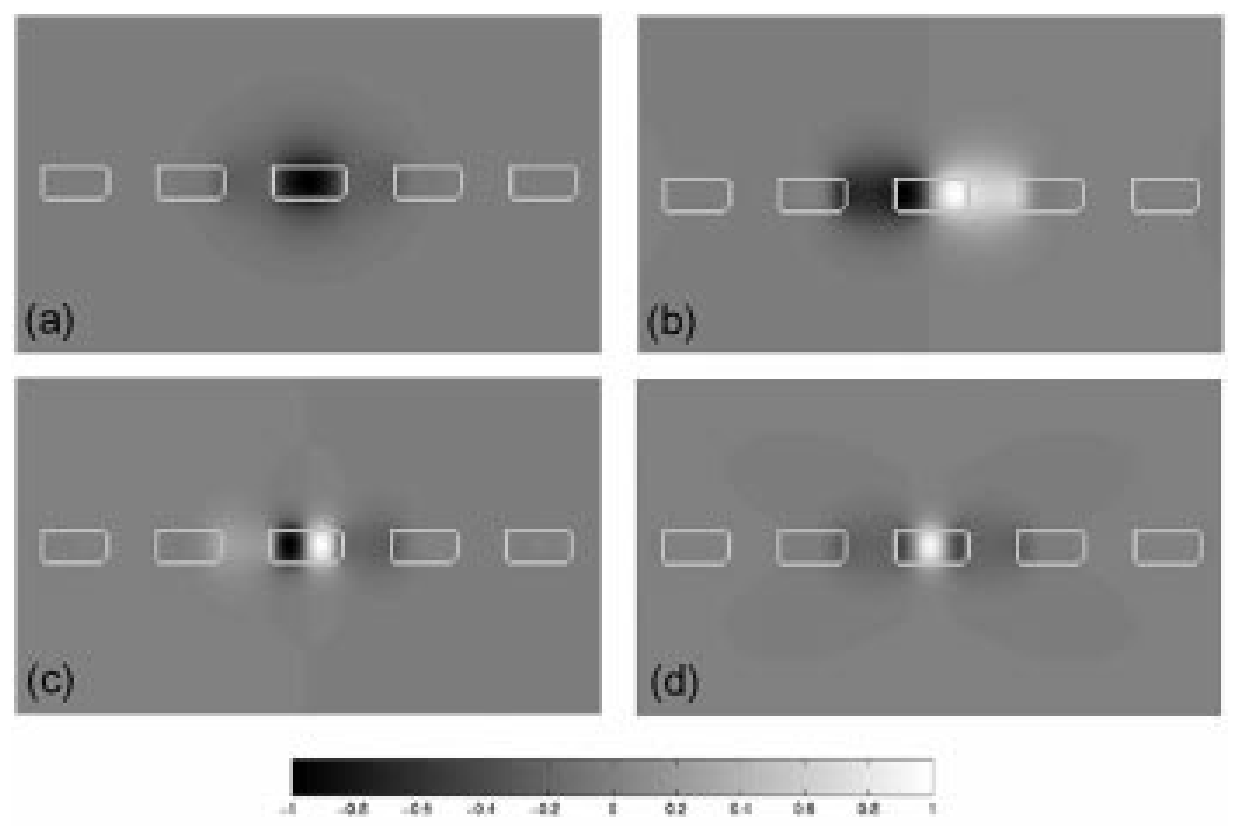

Fig. 7. Distribution of the $B_{z}$ component of the field along the cross section of the triangular-lattice-based waveguide ( $\sigma_{x z z}-$ plane) for different guided modes. Labeling is (mode \#, $\left.k_{y} \cdot(a / \pi), a / \lambda\right)$. (a) (e1, 17/24, 0.191), (b) (e2, 24/24, 0.260), (c) (e4, 21/24, 0.346), and (d) (e5, 21/24, 0.382). Again, all the modes are fundamental in the vertical direction. The core of the waveguide appears as the rectangle in the center of the figure. Intensity scalebar is also shown.

[Fig. 4(b)] tells us that the mode 02 exists in the same frequency range as the $\mathrm{e} 2$, and, therefore, the single-mode operation of waveguide is not possible. However, this problem could be solved by selecting the polarization of the input light, so that we preferentially couple to the e 2 mode. Modes e 3 and e 4 in the second-order gap have different spatial profiles but are very close in frequency. This can be understood by analyzing the properties of the PC slab modes. We observe that the second air band along the $X M$ direction [Fig. 2(a), even modes] is double degenerate, and therefore when pulled down in the bandgap will produce two closely spaced modes e 3 and e 4 .

In Fig. 4(b) we show the dispersion relation for guided modes with vertically odd symmetry (TM-like). Although there is no bandgap for TM-like modes [Fig. 2(a)], light can be localized to the waveguide due to the refractive index contrast, as described above.

In Fig. 5 we show dispersion relations for the guided modes in the waveguide based on the triangular PC lattice. In the case of a triangular lattice waveguide, a single-line defect is formed in the $\Gamma J$ direction [Fig. 1(b)]. Again, $k_{y}(\beta)$ can assume values in the interval $(0, \pi / a)$ that represents $3 / 4$ of the $\Gamma J$ direction. In Fig. 5, we have projected all the bands from Fig. 2(b) to the $(0,3 / 4 * \Gamma J)$ range (gray regions). We see that the triangular-lattice-based waveguide can support several TE-like modes. Two of them, e1 and e2, are index guided and lie below the modes of PC lattice. Other modes are PBG effect guided, and are located in the bandgap. The structure also supports two index guided TM-like modes [Fig. 5(b)]. Since the bandgap is closed in the case of TM-like modes [Fig. 2(b)] there are no PBG effect guided TM-like modes. In Fig. 7 we show field profiles of the $B_{z}$ components along the cross section of the waveguide $\left(\sigma_{x z}\right.$-plane) for several different modes.

Very often it is desirable for a waveguide to operate in the single-mode regime. Although the triangular-lattice-based waveguide is multimode, all PBG-effect guided modes are separated in frequency. Therefore, by working in one frequency range we can excite only one TE-like guided mode at a time. However, it can be observed that leaky modes are present at all bandgap frequencies. Because of that, when coupling light from an external source to the guided mode of the waveguide, we excite these leaky modes as well, and coupling efficiency decreases. Therefore, in order to have good coupling efficiency, we should eliminate leaky modes in the frequency range where guided mode of interest is present. This can be done, for example, by reducing r/a ratio of the PC lattice [26] or by narrowing the waveguide [27]. As in the case of the square-lattice waveguide, both TE-like and TM-like guided modes exist in the frequency range of interest (midgap of the first-order bandgap). The single-mode regime of operation can be achieved in two ways: by selecting the polarization of the input light, or lithographically, by reducing the width of the line defect. By narrowing the waveguide, we can push some of the modes back into the air band and reduce the number of guided modes. In that way, we could increase the cutoff frequency of the TM-like modes while keeping some of the TE-like ones, and achieve a single-mode regime of operation.

In conclusion, we have shown that there are two types of guided modes in both square- and triangular-lattice-based waveguides. In addition, we have shown that guiding can occur for the vertically odd modes (TM-like) due to the effective refractive index contrast, although there is no bandgap for TM-like modes. Index-guided modes (all of the TM-like modes) are not expected to have high transmission around sharp corners in the structures that we describe in the next section. (However, Manolatou et al. [28] have recently proposed and analyzed, in two dimensions, a novel way of light guiding around sharp bends using index guiding in standard ridge waveguides with cavities.) On the other hand, modes 


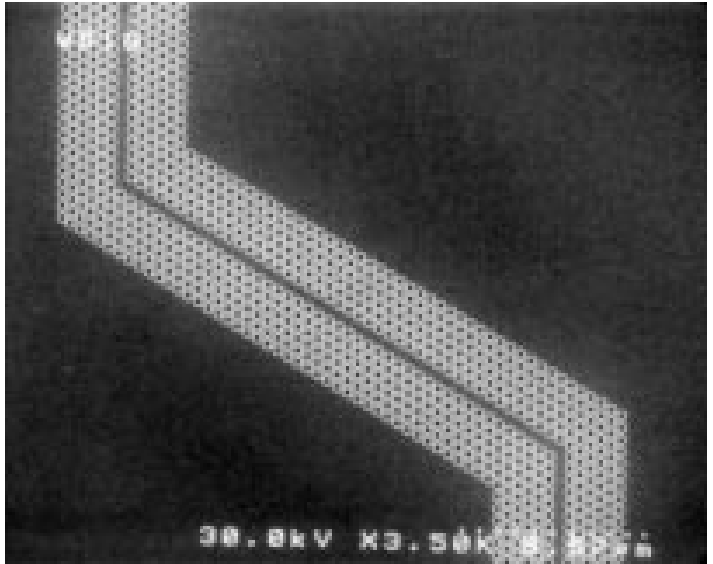

(a)

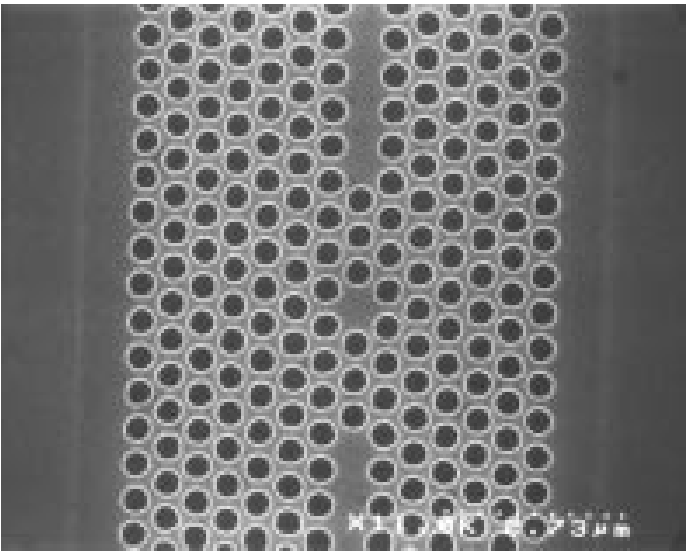

(b)

Fig. 8. (a) Waveguide with two $60^{\circ}$ bends and $25 \mu \mathrm{m}$ bend-to-bend spacing. (b) High- $Q$ cavity filter formed by coupling single-defect cavity to the two waveguide sections.

that are located in the bandgap are expected to have high bend transmission due to the fact that light cannot escape into the photonic crystal, but only can be scattered out in the vertical direction. Recently, transmission around sharp bends in the 3-D structure has been numerically characterized by Chutinan et al. [26].

\section{FABRICATION}

In this section, we describe the fabrication procedure for making photonic crystal waveguides. We have fabricated straight waveguides and waveguides with a different number of $90^{\circ}$ and $60^{\circ}$ bends in square and triangular lattices, respectively. $90^{\circ}$ bends are fabricated with three different lengths of the bend cavity [14]. In the case of two-bends waveguides [Fig. 8(a)] we have fabricated structures with three different bend-to-bend lengths $(25,65$, and $105 \mu \mathrm{m})$. In addition, we have fabricated structures that incorporate different types of cavities coupled to the input and output waveguide [Fig. 8(b)]. These types of structures can be used as high- $Q$ filters but are also very promising candidates for quantum computing applications [29].

Our waveguides were fabricated in silicon-on-insulator (SOI) wafer, as single-crystal silicon is known to be transparent within the interesting 1.3-1.7 $\mu \mathrm{m}$ wavelength range. The fabrication
TABLE II

THE STRUCTURE OF THE WAFER

\begin{tabular}{c|c|c}
\hline Layer Number & Layer Type & Layer Thickness \\
\hline 3 (top) & Si slab & $340 \mathrm{~nm}$ \\
\hline 2 & SiO $_{2}$ & $340 \mathrm{~nm}$ \\
\hline 1 & Si substrate & $\approx 500 \mu \mathrm{m}$ \\
\hline
\end{tabular}

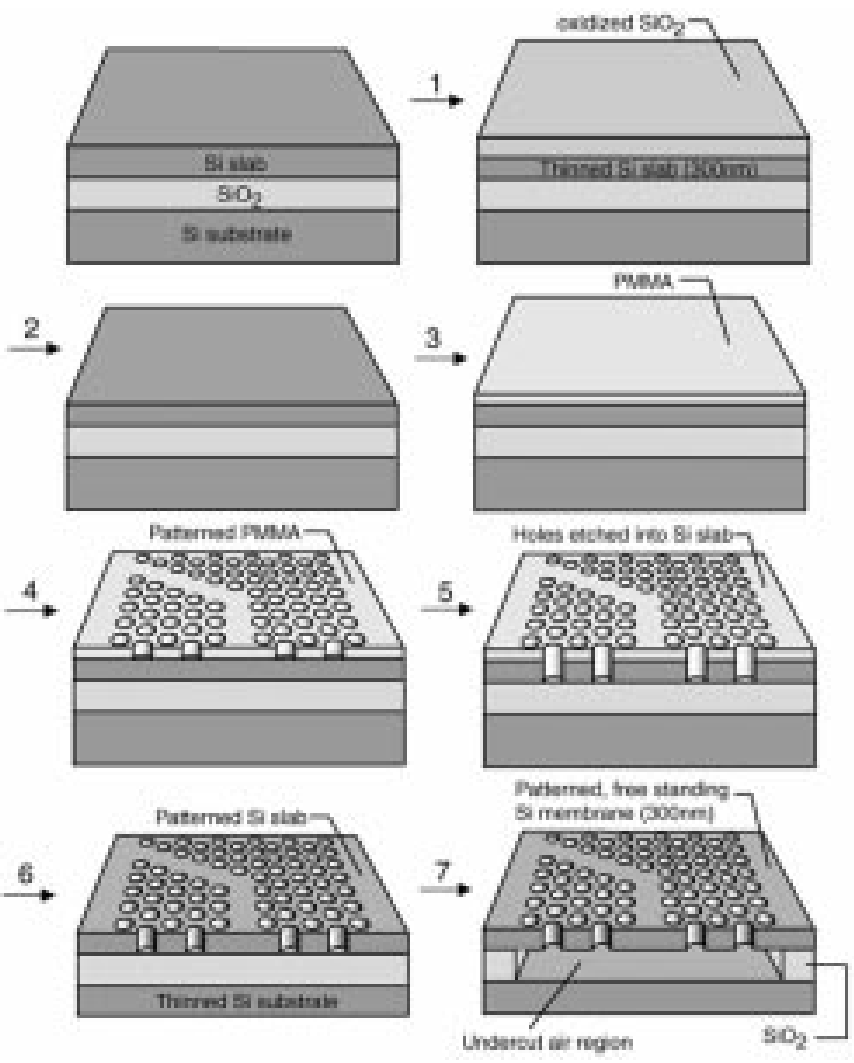

Fig. 9. Fabrication procedure: 1) oxidation and 2) HF dip in order to define the thickness of the Si slab; 3) deposition of PMMA and 4) electron-beam lithography to define patterns in PMMA; 5) CAIBE to transfer patterns into silicon; 6) removal of PMMA and thinning of the substrate; 7) HF dip to remove the $\mathrm{SiO}_{2}$ layer underneath the waveguide in order to improve the vertical confinement of light.

techniques to define microstructures within silicon have been thoroughly researched, and it is possible to obtain large areas of high-quality waveguide slabs of known thickness. The advantage of the procedure described here is that, due to the high etching rate selectivity of polymethylmethacrylate (PMMA) over silicon, no mask amplification procedure is required to define the photonic crystals. Patterns are defined in PMMA using electron-beam lithography, and directly transferred into Si using chemically assisted ion beam etching [30] (CAIBE).

The structure of the wafer is shown in Table II and the whole fabrication procedure is shown in the Fig. 9. As discussed before, the optimal thickness of the Si slab is around $0.182 * \lambda$ for the square and $0.2 * \lambda$ for the triangular lattice. This thickness can be controlled on an SOI wafer by oxidizing the surface of the Si until the desired silicon thickness is obtained.

After oxidation, the formed $\mathrm{SiO}_{2}$ layer was removed from the top by dissolution in hydrofluoric acid. The sample was then prepared for electron-beam lithography. A PMMA, with 


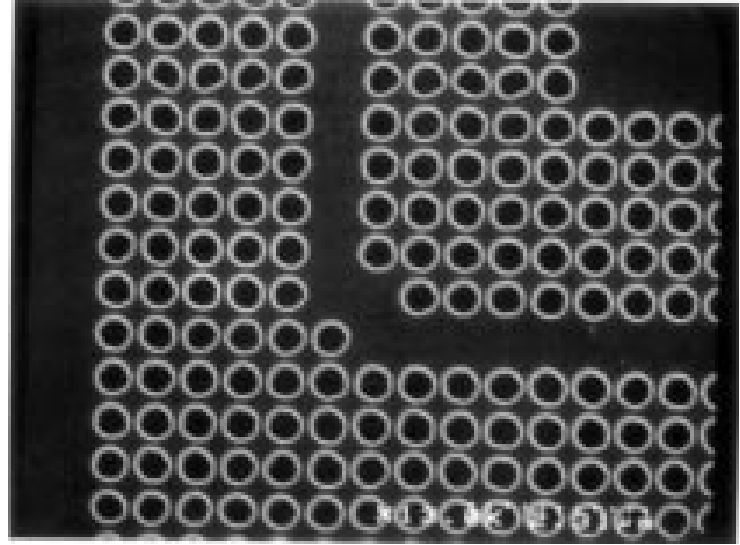

(a)

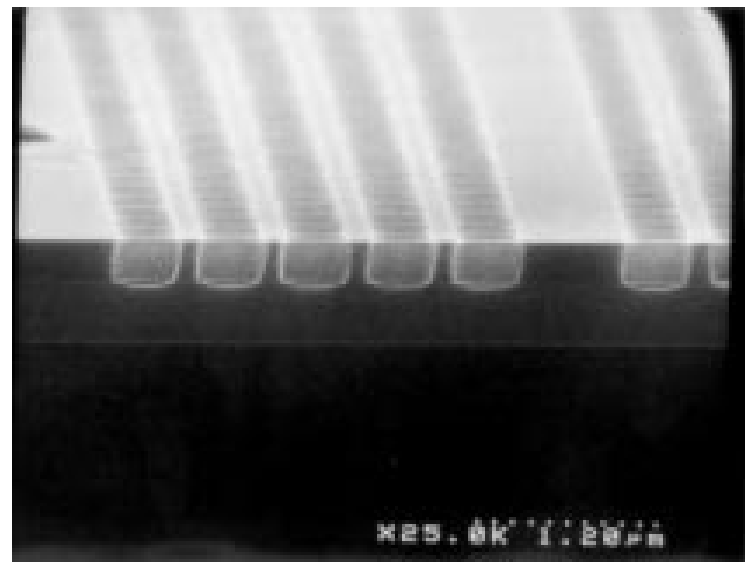

(b)

Fig. 10. (a) SEM micrograph of the $90^{\circ}$ bend in the square lattice after CAIBE (b) SEM micrograph of the cross section of the square lattice waveguide. Layers top-down are $\mathrm{Si}$ slab $(280 \mathrm{~nm}), \mathrm{SiO}_{2}(340 \mathrm{~nm})$, and $\mathrm{Si}$ substrate. The lattice constant is $a \approx 500 \mathrm{~nm}$ and hole radius $r \approx 200 \mathrm{~nm}$.

molecular weight of $950 \mathrm{~K}$, was deposited on the sample by spinning. The sample was then baked on a hot plate at $150{ }^{\circ} \mathrm{C}$ for 45 min. The resulting PMMA thickness was measured to be $150 \mathrm{~nm}$. Electron-beam lithography was performed in a converted Hitachi S-4500 field emission scanning electron microscope operated at $30 \mathrm{kV}$. The $500-\mu \mathrm{m}$ sections of both triangular- and square-lattice photonic crystal waveguides were defined. The exposed PMMA was then developed in a 3:7 mixture of 2-ethoxyethanol and methanol for $30 \mathrm{~s}$ and finally rinsed in IPA for $15 \mathrm{~s}$. Patterns were then transferred through the Si slab using CAIBE with a $1250-\mathrm{V} \mathrm{Ar}{ }^{+}$beam assisted by $\mathrm{XeF}_{2}$ as a reactive gas [Fig. 10(a)]. We measure a 7:1 selectivity of $\mathrm{Si}$ etch rates versus PMMA in our optimized CAIBE system, which enables us to use relatively thin PMMA layers (50 $\mathrm{nm}$ thick) as etch masks. In addition, the directionality of etch was found to be almost vertical, as can be observed in Fig. 10(b). This high selectivity and anisotropy permit us to define high-quality photonic crystal structures within SOI silicon structures. After the CAIBE etching procedure, the PMMA resist was removed from the top of the sample.

In order to be able to couple the light in and out of the waveguide it is necessary to have access to both of its ends. To do so, the samples were cleaved from both sides. Prior to cleaving, the sample was mechanically polished from the back side using

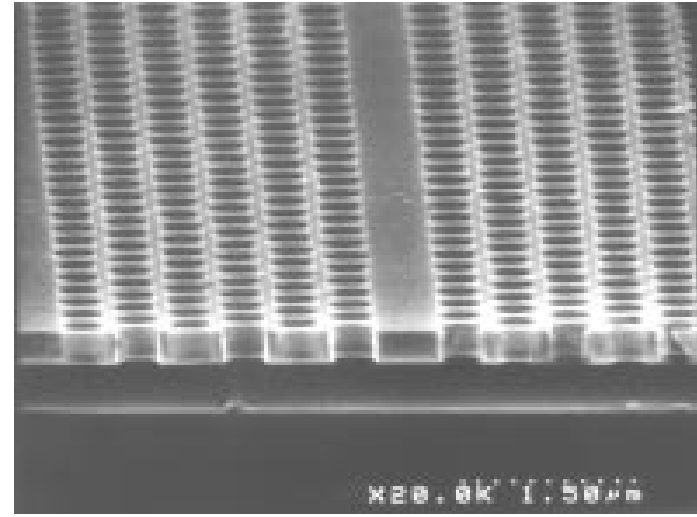

(a)

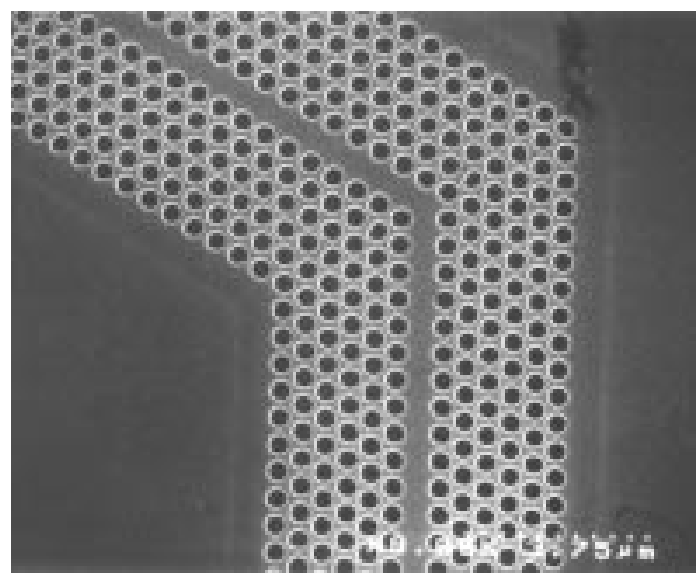

(b)

Fig. 11. (a) SEM micrograph of the cross section of the triangular-structure waveguide suspended in air. Layers top-down are $\mathrm{Si}$ slab/air/Si substrate. (b) SEM micrograph of $60^{\circ}$ bend in the triangular lattice. Area that is undercut extends about $1 \mu \mathrm{m}$ around the waveguide. Parameters of the fabricated structure are $a \approx 530 \mathrm{~nm}, r \approx 208 \mathrm{~nm}$, and $d \approx 300 \mathrm{~nm}$.

$\mathrm{Al}_{2} \mathrm{O}_{3}$ polishing powder, and thinned from 500 to below 100 $\mu \mathrm{m}$. This enabled us to obtain smooth cleaved edges at both sides of the waveguide [Fig. 10(b)] which is very important in order to reduce the insertion losses during optical coupling from a glass fiber to the photonic crystal waveguide.

The final step, undercutting of the waveguide, again required immersion in hydrofluoric acid. This was performed in order to increase the refractive index contrast in the vertical direction and thereby improve the vertical confinement of the light within the slab. In addition, by having the slab surrounded by air at both sides, the structure is made symmetric and its eigenmodes can be characterized by their symmetry properties in the vertical direction. As we have seen, the bandgap is open only in the case of vertically even modes. If we did not remove $\mathrm{SiO}_{2}$ layer, the symmetry would be broken, and the distinction between even and odd modes of the slab destroyed. Formerly odd modes would come into play and possibly close the bandgap [9]. The $\mathrm{SiO}_{2}$ layer underneath the waveguides was removed by dissolution through the ion-etched holes. The fabricated SOI sample was dipped into $49 \% \mathrm{HF}$ for $30 \mathrm{~s}$, and was then rinsed with deionized water for $1 \mathrm{~min}$. This process left the Si waveguide and photonic crystal mirror membrane suspended in the air. Fig. 11 shows the cross section and the top view of a typical photonic crystal waveguide after HF undercutting. 
The design parameters of the fabricated square and triangular structures are summarized in Table I. In the case of square lattice, design parameters of the fabricated structure match well with the designed ones. Fabricated triangular-lattice structure is about 5\% smaller than the designed one. However, due to the wide bandgap of the triangular lattice, this change is not significant. The normalized frequency at $\lambda=1.5 \mu \mathrm{m}$ (for $a=530$ $\mathrm{nm})$ is $a / \lambda=0.353$, still in the bandgap.

\section{CONCLUSION}

We have presented the design and fabrication of photonic crystal waveguides. 3-D FDTD analysis was used to find the eigenmodes of an optically thin slab perforated with 2-D lattice of holes, and single-line defects in both triangular and square PC lattices. We have shown that a single-line defect supports both effective index contrast guided and PBG effect guided modes. In addition, we have shown that the index guiding can occur for the vertically odd modes (TM-like) although the bandgap is not open in that case. We have developed a new fabrication technique for defining these structures in SOI wafers. We have characterized the single-line defect waveguides presented here and we were able to see evidence of guiding of light around $60^{\circ}$ and $90^{\circ}$ corners [20].

\section{ACKNOWLEDGMENT}

The authors gratefully acknowledge many fruitful discussions with O. Painter, R. Lee, and Prof. A. Yariv from Caltech, Pasadena, CA, and D. Nedeljkovic and T. P. Pearsall from Corning, Inc., (France). The authors also wish to thank Prof. Yariv for making his laboratory and polishing and cleaving equipment available to them to conduct these experiments.

\section{REFERENCES}

[1] J. D. Joannopoulos, R. D. Meade, and J. N. Winn, Photonic Crystals. Princeton, NJ: Princeton Univ. Press, 1995.

[2] E. Yablonovitch, "Inhibited spontaneous emission in solid-state physics and electronics," Phys. Rev. Lett., vol. 58, pp. 2059-2062, 1987.

[3] C. C. Cheng and A. Scherer, "Fabrication of photonic band-gap crystals," J. Vac. Sci. Technol. B, vol. 13, pp. 2696-2700, 1995.

[4] S. Noda, N. Yamamoto, M. Imada, H. Kobayashi, and M. Okano, "Alignment and stacking of semiconductor photonic bandgaps by wafer-fusion," J. Lightwave Technol., vol. 17, pp. 1948-1955, 1999.

[5] S. Lin and J. G. Fleming, "A three-dimensional optical photonic crystal," J. Lightwave Technol., vol. 17, pp. 1944-1947, 1999.

[6] T. F. Krauss, R. M. De La Rue, and S. Brand, "Two-dimensional photonic-bandgap structures operating at near infrared wavelengths," $\mathrm{Na}$ ture, vol. 383, pp. 699-702, 1996.

[7] P. R. Villeneuve, S. Fan, S. G. Johnson, and J. D. Joannopoulos, "Three-dimensional photon confinement in photonic crystals of low-dimensional periodicity," Proc. Inst. Elec. Eng.-Optoelectron., vol. 145, pp. 384-390, 1998.

[8] B. D’Urso, O. Painter, J. O'Brian, T. Tombrello, A. Yariv, and A. Scherer, "Modal reflectivity in finite-depth two-dimensional photonic-crystal microcavities," J. Opt. Soc. Amer. B, vol. 15, pp. 1155-1159, 1998.

[9] S. G. Johnson, S. Fan, P. R. Villeneuve, and J. D. Joannopoulos, "Guided modes in photonic crystal slabs," Phys. Rev. B, vol. 60, pp. 5751-5758, 1999.

[10] O. Painter, J. Vučković, and A. Scherer, "Defect modes of a two-dimensional photonic crystal in an optically thin dielectric slab," J. Opt. Soc. Amer. B, vol. 16, pp. 275-285, 1999.

[11] O. Painter, R. K. Lee, A. Yariv, A. Scherer, J. D. O'Brian, P. D. Dapkus, and I. Kim, "Two-dimensional photonic band-gap defect mode laser," Science, vol. 284, pp. 1819-1821, 1999.
[12] D. J. Rippin, K. Lim, G. S. Petrich, P. R. Villeneuve, S. Fan, E. R. Thoen, J. D. Joannopoulos, E. P. Ippen, and L. A. Kolodziejski, "One-dimensional photonic bandgap microcavities for strong optical confinement in $\mathrm{GaAs}$ and $\mathrm{GaAs} / \mathrm{Al}_{x} \mathrm{O}_{y}$ semiconductor waveguides," J. Lightwave Technol., vol. 17, pp. 2152-2160, 1999.

[13] R. D. Meade, A. Devenyi, J. D. Joannopoulos, O. L. Alerhand, D. A. Smith, and K. Kash, "Novel applications of photonic band gap materials: Low-loss bends and high $Q$ cavities," J. Appl. Phys., vol. 75, pp. 4753-4755, 1994.

[14] A. Mekis, J. C. Chen, I. Kurland, S. Fan, P. R. Villeneuve, and J. D. Joannopoulos, "High transmission through sharp bends in photonic crystal waveguides," Phys. Rev. Lett., vol. 77, pp. 3787-3790, 1996.

[15] S. Lin, E. Chow, V. Hietala, P. R. Villeneuve, and J. D. Joannopoulos, "Experimental demonstration of guiding and bending of electromagnetic waves in a photonic crystal," Science, vol. 282, pp. 274-276, 1998.

[16] I. El-Kady, M. M. Sigalas, R. Biswas, and K. M. Ho, "Dielectric waveguide in two-dimensional photonic bandgap materials," J. Lightwave Technol., vol. 17, pp. 2042-2049, 1999.

[17] S. Kuchinsky, D. C. Allan, N. F. Borelli, and J. C. Cotteverte, "3D localization in a channel waveguide in a photonic crystal with $2 \mathrm{D}$ periodicity," Opt. Commun., vol. 175, pp. 147-152, 2000.

[18] T. Baba, N. Fukaya, and J. Yonekura, "Observation of light propagation in photonic crystal optical waveguides with bends," Electron. Lett., vol. 35, pp. 654-655, 1999.

[19] M. Tokushima, H. Kosaka, A. Tomita, and H. Yamada, "Lightwave propagation through a $120^{\circ}$ sharply bent single-line-defect phoonic crystal waveguide," Appl. Phys. Lett., vol. 76, pp. 952-954, 2000.

[20] M. Lončar, D. Nedeljković, J. C. Cotteverte, T. Doll, A. Scherer, J. Gerretsen, and T. P. Pearsall, "Waveguiding at $1500 \mathrm{~nm}$ using photonic crystal structures in silicon on insulator wafers," in Proc. CLEO/QELS, 2000, p. 89.

[21] K. S. Yee, "Numerical solution to initial boundary value problems involving Maxwell's equations in isotropic media," IEEE Trans. Antennas Propagat., vol. AP-14, pp. 302-307, 1966.

[22] A. Taflove, Computational Electrodynamics-The Finite-Difference Time-Domain Method. Boston, MA: Artech House, 1995.

[23] M. Boroditsky, R. Coccioli, and E. Yablonovitch, "Analysis of photonic crystals for light emitting diodes using the finite difference time domain technique," Photonics West, 1998.

[24] G. Mur, "Absorbing boundary conditions for the finite-difference approximation of the time-domain electromagnetic equations," IEEE Trans. Electromagn. Compat., vol. EMC-23, pp. 377-382, 1981.

[25] S. Fan, P. R. Villeneuve, and J. D. Joannopoulos, "Large omnidirectional band gaps in metallodielectric photonic crystals," Phys. Rev. B, vol. 54, pp. 11245-11251, 1996.

[26] A. Chutinan and S. Noda, "Analysis of waveguides and waveguide bends in photonic crystal slabs with triangular lattice," Jpn. J. Appl. Phys., vol. 39, pp. L595-L596, 2000.

[27] M. Lončar, J. Vučković, and A. Scherer, "Modal analysis of waveguides based on a triangular photonic crystal lattice," in LEOS 2000 Annual Meeting, Nov. 2000, to be published.

[28] C. Manolatou, S. G. Johnson, S. Fan, P. R. Villeneuve, H. A. Haus, and J. D. Joannopoulos, "High-density integrated optics," J. Lightwave Technol., vol. 17, pp. 1682-1692, 1999.

[29] J. Vučković, M. Lončar, H. Mabuchi, and A. Scherer, "Photonic crystal cavities for strong coupling between an atom and the cavity field," in LEOS Annual Meeting, Nov. 2000, to be published.

[30] A. Scherer, B. P. Van Der Gaag, K. Kash, H. G. Craighead, and P. Grabbe, "Anisotropic ion beam assisted etching of optical structures in GaAs and InP," Mat. Res. Soc. Symp. Proc., vol. 126, pp. 245-250, 1988.

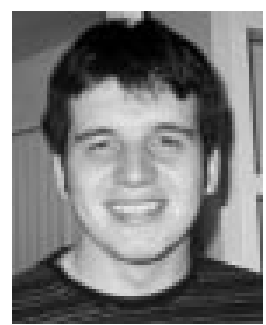

Marko Lončar was born in Dubrovnik, Croatia, in 1974. He received the Dipl. Ing. degree from the Faculty of Electrical Engineering, University of Belgrade, Yugoslavia, in 1997 and the M.S. degree in electrical engineering from the California Institute of Technology, Pasadena, in 1998. Currently, he is working towards the Ph.D. degree in electrical engineering at California Institute of Technology.

His research interests include design and fabrication of photonic crystal devices and ultrasmall device processing techniques. 


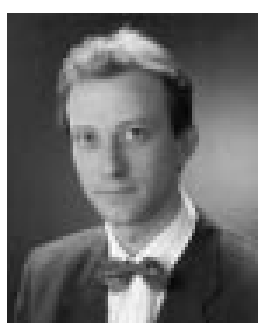

Theodor Doll received the degree in physics from Munich University, Germany, in 1989. He received the Ph.D. degree in electrical engineering from the Munich Bundeswehr University in 1995, where he was subsequently appointed an Assistant Professor for microsystems and nanotechnology.

After a Humboldt Fellowship to the California Institute of Technology, Pasadena, 1998-2000, he is now a Professorate in Solid State Electronics at the Ilmenau Technical University, Germany. His research interests are MEMS, optical electronical nanodevices as well as chemosensors.

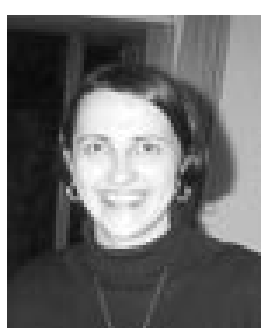

Jelena Vučković was born in Niš Yugoslavia, in 1971. She received the Dipl. Ing. degree from the University of Niš, Faculty of Electronic Engineering, in 1993. In 1997, she received the M.S. degree in electrical engineering from California Institute of Technology, Pasadena, where she is currently working towards the Ph.D. degree.

During 1994 and 1995, she worked as an assistant at the University of Niš and during 1996 as a Researcher at the School of Electrical Engineering of the University of Sydney, Australia. Her research interests include the design and fabrication of optical microcavities and photonic crystal-based optical devices.

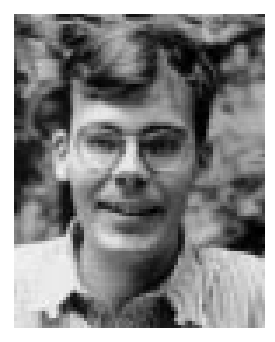

Axel Scherer received the B.S., M.S., and Ph.D. degrees from the New Mexico Institute of Mining and Technology in 1981, 1982, and 1985, respectively.

From 1985 to 1993, he worked in the Quantum Device Fabrication group at Bellcore. Currently, he is Professor of electrical engineering, applied physics, and physics at California Institute of Technology, Pasadena, specializing in device microfabrication. His research interests include design and fabrication of functional photonic, nanomagnetic, and microfluidic devices. 